\title{
Vancomycin Susceptible Enterococcus
}

National Cancer Institute

\section{Source}

National Cancer Institute. Vancomycin Susceptible Enterococcus. NCI Thesaurus. Code C125981.

A non-taxonomic grouping of enterococcus species that are sensitive to vancomycin antibiotics. 